\title{
High-sensitive cardiac Troponin T and exercise stress test for evaluation of angiographically significant coronary disease.
}

\author{
Authors: \\ Joanna Cwikiel MD ${ }^{a, c, d}$, Ingebjørg Seljeflot Professor, PhD ${ }^{a, b, c}$, Morten W Fagerland PhD \\ Kristian Wachtell MD, $\mathrm{PhD}^{\mathrm{e}}$, Harald Arnesen Professor emeritus, MD, PhD ${ }^{\mathrm{a}, \mathrm{c}}$, Eivind \\ Berge MD, PhD ${ }^{\mathrm{b}}$, Arnljot Flaa MD, $\mathrm{PhD}^{\mathrm{b}, \mathrm{d}}$.
}

\author{
Author affiliations: \\ a Center for Clinical Heart Research, Department of Cardiology, Oslo University Hospital \\ Ullevaal, Norway \\ ${ }^{b}$ Department of Cardiology, Oslo University Hospital Ullevaal, Norway \\ c Faculty of Medicine, University of Oslo, Norway \\ ' Section of Cardiovascular and Renal research Oslo University Hospital Ullevaal, Norway \\ e Department of Cardiology, Section for Cardiology Intervention, Division of Cardiovascular \\ and Pulmonary Diseases, Oslo University Hospital, Norway \\ f Oslo Centre for Biostatistics and Epidemiology, Research Support Service, Oslo University \\ Hospital, Oslo, Norway
}

\section{Co-authors e-mail addresses:}

Arnljot Flaa arnljot.flaa@gmail.com

Eivind Berge eivind.berge@medisin.uio.no

Ingebjorg Seljeflot uxinlj@ous-hf.no

Morten Wang Fagerland morten.fagerland@medisin.uio.no

Kristian Wachtell kriwac@ous-hf.no

Harald Arnesen uxhaar@ous-hf.no

\section{Correspondence:}

Joanna Cwikiel

Center for Clinical Heart Research,

Department of Cardiology,

Oslo University Hospital Ullevaal,

PB 4956 Nydalen

0424 Oslo, Norway

E-mail: jcwikiel@gmail.com

Tel: +4722119442

Tweet: Resting and exercise induced rise in hs-cTnT have a predictive value alone, as well as added to a diagnostic EST for diagnosis of highly significant CAD on angiography among patients presenting with symptoms suspective of stable CAD. 


\begin{abstract}
Background: Exercise stress test (EST) has a moderate precision for diagnosis of CAD and could potentially obtain improved accuracy if adding a reliable cardiac biomarker to the test.

Objective: We aimed to investigate resting levels and change in hs-cTnT during EST in patients with and without angiographically significant CAD. Moreover, we intended to explore the additive value of hs-cTnT to EST results in diagnosis of stable CAD. We hypothesized that hs-cTnT would be higher in CAD patients and increase diagnostic precision of EST.

Method: Patients presenting with symptoms of stable CAD, performed a maximal EST on a bicycle ergometer. Venous blood samples were taken at rest and within 5 min postexercise. All patients underwent coronary angiography. Significant CAD was defined as having $\geq 75 \%$ stenosis in one or more segments of the coronary arteries.

Results: Out of the 297 participants, significant CAD was found in 111 (37\%) patients.

Patients with significant CAD compared to without, had higher resting levels of hs-cTnT (median 8.1 vs $5.0 \mathrm{ng} / \mathrm{L}$ ) and no significant difference in exercise-induced change (median 0.5 vs $0.3 \mathrm{ng} / \mathrm{L}$ ), $\mathrm{p}<0.001$ and $\mathrm{p}=0.086$ respectively. Combined resting hs-cTnT with EST had higher predictive value for significant CAD than EST alone, AUC $=0.751$ vs. $\mathrm{AUC}=0.637$. In an adjusted multivariable regression analysis, resting hs-cTnT $>6.0 \mathrm{ng} / \mathrm{L}$ was predictive for having significant CAD, OR 2.55 (CI 95\% 1.40, 4.65 p=0.002).

Conclusion: In patients with suspected stable CAD, hs-cTnT has a predictive value alone, as well as added to a diagnostic EST for CAD.
\end{abstract}

Keywords: Coronary artery disease; coronary angiography; Troponin T; exercise stress test 
Abbreviations

ACS=acute coronary syndrome

$\mathrm{CABG}=$ coronary artery bypass graft

$\mathrm{CAD}=$ coronary artery disease

$\mathrm{EST}=$ exercise stress test

$\mathrm{FFR}=$ fractional flow reserve

$\mathrm{NPV}=$ negative predictive value

$\mathrm{OR}=0$ dds ratio

PPV=positive predictive value

ROC=receiver opertering characteristics

$\mathrm{TnT}=$ Troponin $\mathrm{T}$ 


\section{Introduction}

In contemporary clinical practice, high-sensitive cardiac troponin assays are preferably used for risk stratification and clinical diagnosis of acute coronary syndrome (ACS). In the general population, among patients with chest pain and known stable coronary artery disease (CAD), high-sensitive cardiac Troponin T (hs-cTnT) has recurrently shown to be of prognostic importance (1) (2) (3). Whether ischemia per se, without evident myocardial injury, may cause leakage of troponins is an ongoing debate and different pathophysiological mechanisms have been suggested (4) (5).

To recognize stable CAD, before it evolves into an unstable state, is essential for patients at risk. Although imaging diagnostic techniques are advancing, peri-procedural complications and expenses of these techniques are nevertheless still of concern (6). Exercise stress test (EST) is a cost-effective diagnostic tool and with low risk of complications, however, the test is known to be of suboptimal diagnostic precision for detecting obstructive CAD. Selection of patients for referral to invasive diagnostic procedures remains a challenge for clinicians.

Previously, studies have reported on increased baseline troponin levels in patients with stable CAD compared to those without (5) (7) (8).

Similarly, troponins have been shown to predict obstructive CAD among stable patients (9). Exercise-induced troponin secretion among healthy individuals has been described (10) (11), whereas for exercise-induced change in troponin levels, among patients with suspected CAD without concomitant cardiac disease, sparse data is available and especially using coronary angiography as the diagnostic tool. 
In this study we aimed to investigate resting and exercise-induced change of hs-cTnT in patients with suspected stable CAD, without other known cardiac disease. Furthermore, we intended to explore whether hs-cTnT in combination with the EST would increase sensitivity and specificity of EST for the diagnosis of CAD.

\section{Methods}

\section{$\underline{2.1 \text { Study population }}$}

Patients referred for exercise stress testing or coronary angiography due to symptoms suggestive of CAD, were enrolled in the CADENCE study (clinicaltrials.gov NCT01495091), at the Department of Cardiology, Oslo University Hospital Ullevaal, Oslo Norway. Patients were eligible for the study if they had symptoms indicative of ischemic heart disease, were $\geq 18$ years of age and had a Morise risk score $\geq 9$ points, indicating intermediate to high risk of cardiovascular disease (12). Exclusion criteria were acute coronary syndrome, clinical heart failure, on-going arrhythmia or implanted pacemaker, moderate to severe valvular heart disease, renal insufficiency (S-creatinine $>150$ $\mu \mathrm{mol} / \mathrm{L}$ ), inability to perform exercise testing or coronary angiography. In addition, patients with prior coronary artery bypass graft (CABG) were excluded. All participants gave written informed consent to participate. The study has been conducted in accordance with the Declaration of Helsinki, and the Regional Ethics Committee in South Eastern Health Region in Norway approved the protocol.

A thorough medical history was recorded before inclusion. Prior to exercise testing, a physical examination including blood pressure, weight and waist circumference was performed. Hypertension was defined according to known diagnosis or use of specific 
medication. Previous CAD was defined as previous re-vascularization or myocardial infarction.

\section{$\underline{2.2 \text { Exercise stress test }}$}

EST was performed using an electrical bicycle ergometer (Schiller CS-200 Excellence, Switzerland or Ergoline, Germany) monitored by a physician and nursing staff. Registration of a resting 12-lead ECG was performed before exercise, while continuous 12-lead ECG monitoring using a computerized electrocardiogram was used during the test. According to protocol the initial workload was 30 watts (W) for women and $50 \mathrm{~W}$ for men, with a gradual increase of $10 \mathrm{~W}$ per min and participant maintaining a pedaling rate (cadence) of about 65 rotations per min. Every third minute auscultatory blood pressure was measured, and patients were asked about their perceived exhaustion using Borg scale (13). The EST results were assessed by one physician and $10 \%$ of the test results were controlled by another physician, with $97 \%$ concordance of the results. Patients were exercised to exhaustion, if there were no clinical signs of ischemia that developed prior to reaching a high intensity level. The test was stopped after a recovery time of $5 \mathrm{~min}$. A positive test result was defined as having ST-segment elevation, horizontal or down-sloping ST-segment $>1.0 \mathrm{~mm}(0.1 \mathrm{mV})$ at $60 \mathrm{msec}$ after the J-point and chest pain or discomfort. Reasons for terminating the test were development of suspected pathological ECG changes such as ST-segment elevation, STsegment depression in leads without Q waves, arrhythmias increasing through exercise, chest pain, patients desire to stop the test, insufficient chronotropic response to exercise and inadequate or exaggerated hypertensive response (systolic blood pressure $\geq 250$ mmHg or diastolic blood pressure $\geq 115 \mathrm{mmHg}$. 


\section{$\underline{2.3 \text { Coronary angiography }}$}

All study participants underwent coronary angiography, using the standard Seldinger technique, mostly by using radial artery access.

Coronary angiograms were performed and described by an interventional cardiologist. All angiograms were retrospectively analyzed by one physician and in cases of doubt (20\%), angiograms were re-evaluated by a blinded interventional cardiologist. Both readers met for consensus. The inter-observer variability was $7 \%$. According to a modified version of American Heart Association segmentation of the coronary arteries, 17 segments were scored using Gensini score (14) (15). This score accounts for different degrees of coronary lesions and their location, and also reflects the total atherosclerotic burden of the coronary arteries. Collateral arteries were not taken into account. Significant CAD was defined as having stenosis $\geq 75 \%$ in any coronary segment, while no CAD included patients with non-significant as well as no coronary atherosclerosis.

\subsection{High-sensitive cardiac Troponin T and laboratory methods}

Blood samples were collected at rest prior to exercise for analyses of troponin T as well as for routine analyses, as occur from Table 1. Within 5 min after termination of the stress test, another sample was drawn for troponin T analyses. Blood without additives was collected, and serum for troponin $\mathrm{T}$ analyses was prepared within one hour by centrifugation $2000 \mathrm{xg}$ for $10 \mathrm{~min}$ at room temperature. Samples were frozen at $-80 \mathrm{C}$ until analyzed in one. 
Measurements of hs-cTnT were performed by personnel blinded for the exercise test and the angiography results by use of electrochemical luminescence immunoassays Eleusis Troponin T hs STAT (Roche Diagnostics, Switzerland) on Cobas ${ }^{\circledR}$ e602 instruments (Roche Diagnostics). Lower limit of blank of hs-cTnT was $3 \mathrm{ng} / \mathrm{L}$ and lower limit of detection $5 \mathrm{ng} / \mathrm{L}$. In patients with levels below limit of blank, a cut-off value of $1.5 \mathrm{ng} / \mathrm{L}$ was used for purpose of statistical analyses. Inter-assay coefficient of variation (CV) from the manufacturer is stated to be $7.8 \%$ at the level of $6.5 \mathrm{pg} / \mathrm{mL}$ and $4.8 \%$ at the level of $11.5 \mathrm{pg} / \mathrm{mL}$. In our routine laboratory a CV of $6 \%$ at the level of $13 \mathrm{ng} / \mathrm{L}$ is given. Routine analyses were performed by conventional laboratory methods.

\section{$\underline{2.5 \text { Statistical analyses }}$}

Data was analyzed using IBM SPSS Statistics version 25.0 (IBM, Armonk, USA) and Stata/SE 15 (Stata Corp LLC, College Station, TX, USA). Laboratory values were mainly not normally distributed and are presented with median value and interquartile range (IQR). Continuous data are otherwise presented as mean and standard deviation, and categorical data are presented as numbers (\%). Depending on the distribution of the continuous data either Student T-test or Mann-Whitney U test was used for comparisons between groups, while Wilcoxon signed rank test was used for pairwise comparisons of continuous data. Differences in medians were estimated with quantile (median) regression. For comparison of categorical variables Chi-square test was used. For multiple comparisons, univariable and multivariable logistic regression analyses were performed for binary outcome variables. Spearman's rho was used to estimate the correlation between continuous variables. Receiver operating characteristic (ROC) curves and the area under the curve (AUC) were used to assess discrimination (i.e. the 
combination of sensitivity and specificity). P-values $<0.05$ were considered statistically significant.

\section{Results}

\subsection{Demographic data and coronary angiography}

Of the 327 patients initially enrolled in the study, 15 patients had previously had CABG, 9 patients resigned before completing the study protocol and 6 patients were found to have $\geq 1$ exclusion criteria not seen prior to inclusion. The remaining patients $(n=297)$ constituted the present study sample. Of the total population 111 patients (37\%) were found to have significant CAD, of which 86 patients were re-vascularized. In the group without significant CAD $(<75 \%$ stenosis $) 8$ patients were re-vascularized as lesions were considered clinically significant.

Demographic, clinical and medical characteristics are shown in Table 1, in the total cohort and according to having verified CAD or not.

Risk factors such as smoking (18\%) and diabetes (19\%) were equally distributed between those with significant CAD versus no CAD. Patients with hypertension (67 vs $55 \%, \mathrm{p}=0.045$ ), and males ( 85 vs $53 \%, \mathrm{p}<0.001$ ) were predominantly found among patients with significant CAD compared to no CAD.

History of CAD was more common among patients diagnosed with $\geq 75 \%$ stenosis on coronary angiography ( $39 \%$ vs $25 \%, \mathrm{p}=0.011$ ). This is also illustrated by a significantly higher number of patients with significant CAD being treated with aspirin, statins or beta blockers ( $\mathrm{p}=0.010$ for all) compared to those without CAD (Table 1). Total 
cholesterol was lower among patients with significant CAD, reflected by higher number of patients treated with statins in this group.

Categorization of patients that were re-vascularized $(n=94)$ versus not $(n=203)$ showed no significant differences in the frequencies of smoking, diabetes or previous history of CAD (data not shown).

\section{$\underline{3.2 \text { Exercise performance }}$}

Mean exercise duration in the total population was 10:05 \pm 3:46 min and exercise capacity $138 \pm 43 \mathrm{~W}$ with non-significant differences between the two groups (Supplementary Table 1). Positive EST results were found in 59 (53\%) patients with significant CAD and in $48(26 \%)$ patients with non-significant CAD, yielding a positive predictive value (PPV) of $55 \%$ and a negative predictive value (NPV) of $73 \%$ (Supplementary Table 2). Of patients with negative test results ( $\mathrm{n}=190), 52$ patients $(47 \%)$ were found to have significant CAD. Maximal heart rate was significantly lower in patients with significant CAD (132 \pm 20 beats per min (bpm)), compared to those with non-significant CAD (145 $\pm 20 \mathrm{bpm})(\mathrm{p}=0.002)$. Mean metabolic equivalent (MET) was also significantly lower in patients with significant CAD $6.6 \pm 2$ vs $7.0 \pm 2$ (p=0.033). Interpretation of stress test results was performed by a clinician blinded for the angiography results.

\section{$\underline{\text { 3.3 Circulating high-sensitive cardiac Troponin T }}$}

Resting, post-exercise and change in levels of hs-cTnT in the total population and according to having significant CAD or not, are presented in Table 2. In 
patients with significant CAD compared to those without, significantly higher resting levels as well as post-exercise levels, were observed (both $\mathrm{p}<0.001$ ).

During exercise there was a significant increase in hs-cTnT ( $\Delta \mathrm{hs}-\mathrm{cTnT})$ in the total population, $\mathrm{p}<0.001$. In patients with significant CAD compared to those without, $\Delta$ hscTnT had a numerically higher increase during exercise, however this was not significant (0.5 ng/L vs $0.3 \mathrm{ng} / \mathrm{L}) \mathrm{p}=0.086$ (Table 2 ).

In $25(8.4 \%)$ of the patients, hs-cTnT levels at rest were found above the upper normal reference limit ( $\geq 14 \mathrm{ng} / \mathrm{L}$ ), of which 19 were found to have significant CAD.

ROC analyses of resting hs-cTnT for predicting CAD are shown in Figure 1. EST had an AUC $=0.64(95 \%$ CI $0.57,0.70 \mathrm{p}<0.001)$ for predicting significant CAD. Resting hs-cTnT had an AUC=0.73 (95\% CI 0.67, 0.79, p<0.001), whereas AUC for $\Delta$ hs-cTnT was 0.56 (95\% CI 0.49, 0.63, p=0.088). Combining resting hs-cTnT with EST increased predictive utility for significant CAD giving an AUC of 0.75 (95\% CI $0.69,0.81, \mathrm{p}<0.001)$ and an AUC of 0.68 (95\% CI 0.62, 0.75, p<0.001) when adding $\Delta$ hs-cTnT. AUC of EST alone was inferior to resting hs-cTnT ( $\mathrm{p}=0.019)$ as well as to resting hs-cTnT combined with EST $(\mathrm{p}<0.001)$

Correlations between hs-cTnT and Gensini score revealed the strongest correlation between resting hs-cTnT r=0.444 (95\% CI $0.35,0.53)$. Whereas the correlation to $\Delta$ hscTnT was $\mathrm{r}=0.130(95 \%$ CI $0.06,0.28)$.

In an univariable logistic regression analysis $\Delta$ hs-cTnT showed an Odds Ratio (OR) of $1.02(95 \%$ CI 0.94, $1.12 \mathrm{p}=0.625)$ for having CAD. $\Delta$ hs-cTnT was divided into quartiles, 
showing the upper quartile ( $\Delta$ hs-cTnT $\geq 0.8 \mathrm{ng} / \mathrm{L}$ ) to have an OR 1.99 (95\% CI 1.04, 3.84 $\mathrm{p}=0.039$ ) for having $\mathrm{CAD}$ compared to the three lower quartiles. In a multivariable regression analysis adjusting for age, sex, previous CAD, total cholesterol, creatinine, positive exercise test, change in systolic blood pressure and in heart rate during exercise neither $\Delta$ hs-cTnT as a continuous variable nor the upper quartile were associated with having CAD (data not shown).

In an univariable logistic regression model, resting hs-cTnT had an OR of 1.13 (95\% CI 1.07, $1.20 \mathrm{p}<0.001$ ) for having significant CAD. When adjusting for the covariates age, sex, resting systolic blood pressure, resting heart rate, previous $\mathrm{CAD}$, total cholesterol and creatinine, the OR was $1.05(95 \%$ CI $1.00,1.10 \mathrm{p}=0.050)$.

Resting hs-cTnT was further divided into quartiles. We found the upper two quartiles (above the median) to predict CAD significantly, also after adjustment for potential covariates. Therefore, further analyses were performed for the median level i.e. below or above $6.0 \mathrm{ng} / \mathrm{L}$ of resting hs-cTnT (Table 3). Having hs-cTnT above the median, positive predictive value (PPV) was 71\% while negative predictive value (NPV) was 63\%, for significant CAD (Supplementary Table 2). Univariable analysis showed an increasing risk for significant CAD with levels of resting hs-cTnT above the median value, OR 4.19 (95\% CI 2.13, $6.95 \mathrm{p}<0.001)$. When adjusting for above mentioned covariates, results remained highly significant, OR 2.55 (95\% CI 0.1.40, 4.65 p=0.002).

Combining EST results with levels of resting hs-cTnT above and below the median are presented in Supplementary Figure 1. In the group with negative EST results, patients who had levels of resting hs-cTnT above the median ( $>6.0 \mathrm{ng} / \mathrm{L})$ were found to have higher incidence of significant coronary stenosis compared to those with hs-cTnT below 
the median $(\mathrm{p}<0.001)$. Also, in patients with positive EST results, those who had higher levels of hs-cTnT had a higher number of patients with significant CAD $(\mathrm{p}<0.001)$.

\section{$\underline{4 . \text { Discussion }}$}

In the present study of 297 participants with suspected CAD, we found significantly higher levels of hs-cTnT at rest and during exercise stress test in patients with angiographically verified CAD. Moreover, resting hs-cTnT combined with EST results, had a better discriminatory value, than that of EST alone, for predicting angiographically significant CAD. In fact, measurement of resting value of hs-TnT alone seems to discriminate angiographically significant CAD better than EST.

To our knowledge, this is the largest study reporting on high-sensitive cardiac Troponin T combined with EST in diagnosis of stable CAD among patients without other known cardiac conditions and using coronary angiography as the diagnostic modality in the total population.

Troponin release due to exercise-induced ischemia has been of central focus lately. Among published data, evidently higher levels of troponins are found in patients with CAD and exercise-induced ischemia than those without (16) (17). However, whether a clinical implication of these findings could be beneficial has, however, not been thoroughly explored.

Different pathobiological derivatives of cardiac troponins have been suggested. Besides release from myocyte necrosis, normal cell turnover and increased cell membrane 
permeability have been proposed as potential secretory mechanisms $(18,19)$. Exercise induced increase may also derive from a stretch-responsive mediated transport of troponins from viable cardiomyocytes to the circulation (20). In our population, the highly significant correlation between Gensini score with increasing levels of resting troponin $\mathrm{T}$, may additionally, to the above mentioned derivatives, be due to microembolization of particles from existing atherosclerosis (21).

Exercise stress test, regardless of the tests low accuracy, is largely applied in the diagnostic investigation of stable CAD (22). In our study, the exercise-induced change in circulating troponin levels was not shown to be an esteemed measure to be applied for diagnosis of significant CAD. However, we showed that adding resting hs-cTnT levels to the stress test improves diagnostic utility for CAD, verified by coronary angiography. Patients presenting with CAD suggestive symptoms and with positive stress test are likely to be referred for further imaging diagnostics. However, among those with negative test results, about one third of patients, have been reported likely to have significant CAD (23). Our results, with resting hs-cTnT levels $>6.0 \mathrm{ng} / \mathrm{L}$ alone, independently of EST results and other confounding factors, being associated with the presence of $\mathrm{CAD}$, implies the favorable value of troponin validation in patients with negative exercise stress test.

When defining CAD in our population, we chose a strict percentage of luminal stenosis level, $\geq 75 \%$ in any coronary segment. This definition seems to correlate well with number of patients who were in need of re-vascularization, with only 8 patients without CAD, according to our definition, who required re-vascularization. We therefore assume 
that only a small number of patients, with truly significant CAD, were inappropriately categorized.

Previous findings of increasing resting hs-cTnT levels with severity of CAD, diagnosed by different cardiac imaging techniques, have been reported on (24) (25) (26). In these studies, hs-cTnT above $5.7 \mathrm{pg} / \mathrm{mL}$ was found to reflect severe coronary lesions $(>70 \%$ stenosis) as well as good sensitivity for myocardial ischemia, which corresponds to our findings. Likewise, Magioni et al., found hs-cTnT $>6 \mathrm{ng} / \mathrm{L}$ to be an independent risk factor for CAD in patients with low risk for CAD (27). In a recent publication, Brzezinski et al explored resting troponin levels in relation to exercise stress test in a large cohort of healthy individuals (28). Although their findings propose an insignificant relation of troponin levels and positive or negative stress test results, this healthy and supposedly asymptomatic population is generally not of concern in clinical practice. In our cohort, with patients at risk of CAD, experiencing angina equivalent symptoms and two thirds of patients with negative stress test, reflects a genuine clinically challenging scenario. In this setting, detection of troponin T levels $>6.0 \mathrm{ng} / \mathrm{L}$ might guide the clinicians' decision of further diagnostic referral. Although, it has to be taken into consideration, that hscTnT assays have higher coefficients of variation for lower detectable levels. Also, to be taken into account, are possible sex-specific cut-off levels of troponins for detection of CAD (29).

With latest European guidelines for rule-in and rule-out troponin measurements after 0$1 \mathrm{~h}$ in patients without ST-segment elevation myocardial infarction, assessment of shortterm troponin release in stable CAD might also be of importance (30). However, time point for assessment of exercise induced troponin elevations may be debated. Axelsson 
et al. found higher baseline and post-exercise troponin T levels, with a peak release $6 \mathrm{~h}$ after exercise, in patients with CAD compared to controls (31). Our study was designed to be clinically applicable, and since previous reports suggest an immediate troponin release, we found immediate blood sampling post-exercise to be best suitable for potential clinical practice (32) (33).

\section{$\underline{\text { 5. Limitations }}$}

Several considerations concerning circulating troponin levels should be accounted for. As we discussed previously, time point for assessment of exercise induced troponin elevations may be questioned as we did not collect blood samples at time points beyond 5 min post-exercise, why we may have missed a later rise in troponin increment. As well the know low precision of the hs-cTnT assay at low concentration is a central limitation. Neither did we evaluate cardiac Troponin I and it remains to be established if similar release patterns exist for this biomarker.

Secondarily, the higher troponin levels in our patients with verified CAD may as well be due to other subclinical conditions such as paroxysmal arrhythmias or perimyocarditis. Since patients were clinically stable without symptoms or signs of acute disease, minor probability exists that they would suffer from pulmonary embolism, acute heart failure or other acute conditions leading to troponin release. However, we did not have echocardiography examinations performed for our population and above mentioned conditions and also structural ventricular disease may not be completely ruled out.

Another limitation is lack of FFR measurements or other intravascular imaging techniques in most of our patients, possibly underestimating degree of stenosis. With 
our limit of significant CAD of $\geq 75 \%$, amount of patients with CAD may have been underreported since 8 patients in the no-significant CAD group were re-vascularized. Symptom severity was not graded using grading scales such as CCS (Canadian Cardiovascular Society) classification of angina, which could have been a useful measure for our patient population.

\section{Conclusion}

In our patients with suspected stable CAD and no other known concomitant cardiac disease, resting hs-cTnT alone as well as when added to a diagnostic exercise stress test, had a significant predictive value for angiographically verified significant CAD. Hs-cTnT was found related to extent of coronary artery disease and a cut-off level of $6.0 \mathrm{ng} / \mathrm{L}$ may be used to differentiate between patients with negative and positive exercise stress test results.

\section{$\underline{\text { 7. Acknowledgement }}$}

The authors thank Department of Cardiology and Section for Cardiology Intervention at Oslo University Hospital, for their support during patient inclusion. We would like to thank Vibeke Kjær and Charlotte Holst Hansen, for their help during patient inclusion. Sissel Aakra and Vibeke Bratseth at Center for Clinical Heart Research, as well as Department of Medical Biochemistry at Oslo University Hospital Rikshospitalet, are acknowledged for laboratory assistance. Miriam Langseth, contributed to electrocardiography analyses. 


\section{Declarations}

\section{$\underline{8.1 \text { Ethics approval and consent to participate }}$}

All participants have given written informed consent to participate. The study has been conducted in accordance with the Declaration of Helsinki, and the Regional Ethics Committee in South Eastern Health Region in Norway approved the protocol.

\subsection{Conflict of interest}

The authors declare that they have no competing interests.

\section{$\underline{8.3 \text { Financial support }}$}

This study was financially supported by University of Oslo and the Stein Erik Hagen Foundation for Clinical Heart Research, Oslo, Norway.

\section{$\underline{\text { 8.4 Authors contributions }}$}

J. Cwikiel was involved in patient inclusion, interpretation of clinical and laboratory data, drafting and finalizing the manuscript. A. Flaa, E. Berge and I. Seljeflot were involved in designing and planning the study, interpreted data and intellectually contributed to drafting and discussion of the manuscript. K. Wachtell contributed to planning analysis of angiograms and interpretation of angiography data. M. Fagerland contributed to statistical analysis and discussion. H. Arnesen was involved in designing the study and in discussion of the manuscript. 


\section{References}

1. de Lemos JA, Drazner MH, Omland T et al. Association of troponin T detected with a highly sensitive assay and cardiac structure and mortality risk in the general population. JAMA. 2010;304:2503-2512.

2. Roos A, Bandstein N, Lundbäck M et al. Stable High-Sensitivity Cardiac Troponin T Levels and Outcomes in Patients With Chest Pain. J Am Coll Cardiol. 2017;70:2226-2236.

3. Omland T, de Lemos JA, Sabatine MS et al. A sensitive cardiac troponin T assay in stable coronary artery disease. N Engl J Med. 2009;361:2538-2547.

4. Orsini E, Caravelli P, Dini FL et al. High sensitivity cardiac troponins: Can they help in diagnosing myocardial ischaemia. Eur Heart J Acute Cardiovasc Care. 20172048872617700868.

5. Puelacher $\mathrm{C}$, Wagener M, Honegger U et al. Combining high-sensitivity cardiac troponin and B-type natriuretic peptide in the detection of inducible myocardial ischemia. Clin Biochem. 2018;52:33-40.

6. Smulders MW, Kietselaer BL, Schalla $\mathrm{S}$ et al. Acute chest pain in the high-sensitivity cardiac troponin era: A changing role for noninvasive imaging. Am Heart J. 2016;177:102-111.

7. Hammadah M, Al Mheid I, Wilmot K et al. Association Between High-Sensitivity Cardiac Troponin Levels and Myocardial Ischemia During Mental Stress and Conventional Stress. JACC Cardiovasc Imaging. 2018;11:603-611.

8. Beatty $\mathrm{AL}, \mathrm{Ku}$ IA, Christenson RH et al. High-sensitivity cardiac troponin T levels and secondary events in outpatients with coronary heart disease from the Heart and Soul Study. JAMA Intern Med. 2013;173:763-769.

9. Adamson PD, Hunter A, Madsen DM et al. High-Sensitivity Cardiac Troponin I and the Diagnosis of Coronary Artery Disease in Patients With Suspected Angina Pectoris. Circ Cardiovasc Qual Outcomes. 2018;11:e004227.

10. Middleton N, Shave R, George K et al. Impact of repeated prolonged exercise bouts on cardiac function and biomarkers. Med Sci Sports Exerc. 2007;39:83-90.

11. Skadberg $\varnothing$, Kleiven $\varnothing$, Bjørkavoll-Bergseth $\mathrm{M}$ et al. Highly increased Troponin I levels following high-intensity endurance cycling may detect subclinical coronary artery disease in presumably healthy leisure sport cyclists: The North Sea Race Endurance Exercise Study (NEEDED) 2013. Eur J Prev Cardiol. 2017;24:885-894.

12. Morise AP, Haddad WJ, Beckner D. Development and validation of a clinical score to estimate the probability of coronary artery disease in men and women presenting with suspected coronary disease. Am J Med. 1997;102:350-356.

13. Borg GA. Psychophysical bases of perceived exertion. Med Sci Sports Exerc. 1982;14:377-381.

14. Austen WG, Edwards JE, Frye RL et al. A reporting system on patients evaluated for coronary artery disease. Report of the Ad Hoc Committee for Grading of Coronary Artery Disease, Council on Cardiovascular Surgery, American Heart Association. Circulation. 1975;51:5-40.

15. Gensini GG. A more meaningful scoring system for determining the severity of coronary heart disease.[letter]. Am J Cardiol 1983;51(3):606.

16. Sabatine MS, Morrow DA, de Lemos JA et al. Detection of acute changes in circulating troponin in the setting of transient stress test-induced myocardial ischaemia using an ultrasensitive assay: results from TIMI 35. Eur Heart J. 2009;30:162-169. 
17. Lee G, Twerenbold R, Tanglay Y et al. Clinical benefit of high-sensitivity cardiac troponin I in the detection of exercise-induced myocardial ischemia. Am Heart J. 2016;173:8-17.

18. Hammarsten $\mathrm{O}$, Mair J, Möckel M et al. Possible mechanisms behind cardiac troponin elevations. Biomarkers. 2018;23:725-734.

19. White HD. Pathobiology of troponin elevations: do elevations occur with myocardial ischemia as well as necrosis. J Am Coll Cardiol. 2011;57:2406-2408.

20. Shave R, Baggish A, George K et al. Exercise-induced cardiac troponin elevation: evidence, mechanisms, and implications. J Am Coll Cardiol. 2010;56:169-176.

21. Korosoglou G, Lehrke S, Mueller D et al. Determinants of troponin release in patients with stable coronary artery disease: insights from CT angiography characteristics of atherosclerotic plaque. Heart. 2011;97:823-831.

22. Task FM, Montalescot G, Sechtem U et al. 2013 ESC guidelines on the management of stable coronary artery disease: the Task Force on the management of stable coronary artery disease of the European Society of Cardiology. Eur Heart J. 2013;34:2949-3003.

23. Gibbons RJ, Balady GJ, Bricker JT et al. ACC/AHA 2002 guideline update for exercise testing: summary article. A report of the American College of Cardiology/American Heart Association Task Force on Practice Guidelines (Committee to Update the 1997 Exercise Testing Guidelines). J Am Coll Cardiol. 2002;40:1531-1540.

24. Laufer EM, Mingels AM, Winkens MH et al. The extent of coronary atherosclerosis is associated with increasing circulating levels of high sensitive cardiac troponin $\mathrm{T}$. Arterioscler Thromb Vasc Biol. 2010;30:1269-1275.

25. Ahmed W, Schlett CL, Uthamalingam S et al. Single resting hsTnT level predicts abnormal myocardial stress test in acute chest pain patients with normal initial standard troponin. JACC Cardiovasc Imaging. 2013;6:72-82.

26. Ndrepepa G, Braun S, Schulz S et al. High-sensitivity troponin T level and angiographic severity of coronary artery disease. Am J Cardiol. 2011;108:639-643.

27. Magnoni M, Masson S, Andreini D et al. Usefulness of High-Sensitivity Cardiac Troponin $\mathrm{T}$ for the Identification of Outlier Patients With Diffuse Coronary Atherosclerosis and Low-Risk Factors. Am J Cardiol. 2016;117:1397-1404.

28. Brzezinski RY, Fisher E, Ehrenwald M et al. Elevated high-sensitive troponin $\mathrm{T}$ in negative stress test individuals. Eur J Clin Invest. 2018;48:e12930.

29. Mueller D, Puelacher C, Honegger U et al. Direct Comparison of Cardiac Troponin T and I Using a Uniform and a Sex-Specific Approach in the Detection of Functionally Relevant Coronary Artery Disease. Clin Chem. 2018;64:1596-1606.

30. Roffi M, Patrono C, Collet JP et al. 2015 ESC Guidelines for the management of acute coronary syndromes in patients presenting without persistent ST-segment elevation: Task Force for the Management of Acute Coronary Syndromes in Patients Presenting without Persistent ST-Segment Elevation of the European Society of Cardiology (ESC). Eur Heart J. 2016;37:267-315.

31. Axelsson A, Ruwald MH, Dalsgaard M et al. Serial measurements of high-sensitivity cardiac troponin $\mathrm{T}$ after exercise stress test in stable coronary artery disease. Biomarkers. 2013;18:304-309.

32. Liebetrau C, Gaede L, Dörr O et al. High-sensitivity cardiac troponin T and copeptin assays to improve diagnostic accuracy of exercise stress test in patients with suspected coronary artery disease. Eur J Prev Cardiol. 2015;22:684-692.

33. Mouridsen MR, Sajadieh A, Carlsen CM et al. Troponin T and N-terminal pro B-Type natriuretic peptide and presence of coronary artery disease. Scand J Clin Lab Invest. 2015;75:204-212. 


\section{Legend to tables and figures.}

\section{Table 1.}

Baseline characteristics for the total population and for patients without and with CAD. $\mathrm{P}$-values reflect differences between patients without and with significant CAD. Categorical variables are shown with number of patients (\%). Age is presented with mean (range). BMI, Morise score, blood pressure and heart rate are presented with mean \pm standard deviation. Laboratory results are presented with median (IQR).

\section{Supplementary Table 1.}

Exercise stress test results for the total population and for patients without and with significant CAD. Variables are presented with mean \pm standard deviation. Positive exercise test results are given in number of patients (\%). P-values reflect differences between patients without and with CAD.

\section{Supplementary table 2.}

Positive and negative predictive values for exercise stress test and median of resting hscTnT. EST=exercise stress test results. Median hs-cTnT=median resting hs-cTnT (6.0 ng/L). PPV=positive predictive value. NPV=negative predictive value.

\section{Table 2.}

Hs-cTnT levels measured pre- and post-exercise and the change during exercise in the total population and in between patients without and with CAD. Median (IQR) and estimated median difference between significant CAD and non-significant CAD groups with 95\% confidence interval and p-values.

\section{Table 3.}

Univariable and multivariable logistic regression models with median of hs-cTnT at rest as a categorical variable, and clinical factors associated with having significant CAD (dependent variable).

\section{Figure 1.}

Receiver operating characteristic curves for significant CAD and resting hs-cTnT, alone or combined with positive exercise stress test results. Values presented in AUC for all analyses.

\section{Supplementary figure 1.}

Percentage of patients with significant CAD in groups of positive and negative exercise stress test results and with levels of hs-cTnT above and below $6.0 \mathrm{ng} / \mathrm{L}$. P-values represent differences of patients with CAD in groups of exercise test results. 
Table 1. Baseline characteristics.

\begin{tabular}{|c|c|c|}
\hline Overall $(n=297)$ & Non-sign. CAD & Sign. CAD \\
\hline & $(n=186)$ & $(n=111)$ \\
\hline
\end{tabular}

\begin{tabular}{|c|c|c|c|c|}
\hline Age (years) & $62(40-87)$ & $61(40-87)$ & $64(42-84)$ & 0.002 \\
\hline Sex (male \%) & $192(65)$ & $98(53)$ & $94(85)$ & $<0.001$ \\
\hline Body Mass Index $\left(\mathrm{kg} / \mathrm{m}^{2}\right)$ & $27 \pm 4$ & $27 \pm 4$ & $28 \pm 4$ & 0.192 \\
\hline Diabetes (\%) & $56(19)$ & $32(17)$ & $24(22)$ & 0.346 \\
\hline Hypertension (\%) & $176(59)$ & $102(55)$ & $74(67)$ & 0.045 \\
\hline Smoking (current) (\%) & $53(18)$ & $38(20)$ & $15(14)$ & 0.132 \\
\hline Previous revasc/MI (\%) & $89(30)$ & $46(25)$ & $43(39)$ & 0.011 \\
\hline Morise score & $15 \pm 3$ & $14 \pm 3$ & $15 \pm 2$ & $<0.001$ \\
\hline Aspirin (\%) & $214(72)$ & $119(64)$ & $95(86)$ & $<0.001$ \\
\hline Statin(\%) & $212(71)$ & $123(66)$ & $89(80)$ & 0.010 \\
\hline ACE/ARB (\%) & $108(36)$ & $63(34)$ & $45(41)$ & 0.248 \\
\hline Beta blocker (\%) & $123(41)$ & $64(34)$ & $59(53)$ & 0.002 \\
\hline Ca blocker (\%) & $48(16)$ & $23(12)$ & $25(23)$ & 0.021 \\
\hline Creatinine $(\mu \mathrm{mol} / \mathrm{L})$ & 79 (69-89) & $76(67-85)$ & $83(75-94)$ & $<0.001$ \\
\hline Total cholesterol (mmol/L) & $4.6(3.9-5.6)$ & $4.8(3.9-5.8)$ & $4.3(3.7-5.3)$ & 0.016 \\
\hline LDL (mmol/L) & $2.6(2.06-3.49)$ & $2.6(2.11-3.55)$ & $2.5(1.95-3.36)$ & 0.140 \\
\hline HDL (mmol/L) & $1.3(1.08-1.77)$ & $1.5(1.16-1.85)$ & $1.3(1.02-1.53)$ & 0.001 \\
\hline Resting SBP (mmHg) & $136 \pm 20$ & $133 \pm 20$ & $141 \pm 21$ & 0.002 \\
\hline Resting DBP (mmHg) & $83 \pm 11$ & $82 \pm 11$ & $84 \pm 11$ & 0.098 \\
\hline Resting HR (bpm) & $69 \pm 12$ & $71 \pm 12$ & $67 \pm 12$ & 0.007 \\
\hline
\end{tabular}


Table 2. Hs-cTnT levels in total population and in patients without or with CAD.

\begin{tabular}{lcccc} 
& $\begin{array}{c}\text { Overall (n=297) } \\
\text { Median (IQR) }\end{array}$ & $\begin{array}{c}\text { Non-sign. CAD (n=186) } \\
\text { Median (IQR) }\end{array}$ & $\begin{array}{c}\text { Sign. CAD (n=111) } \\
\text { Median (IQR) }\end{array}$ & $\begin{array}{c}\text { Estimated median differance } \\
\text { Sign. CAD - Non-sign. CAD } \\
(95 \% \text { CI); P-value }\end{array}$ \\
\hline Rest hs-cTnT (ng/L) & $6.0(4.3-8.8)$ & $5.0(3.4-7.7)$ & $8.1(5.5-11.3)$ & $3.1(95 \% \mathrm{CI}: 2.18,3.98) ;<0.001$ \\
Post hs-cTnT (ng/L) & $6.6(4.5-9.4)$ & $5.4(3.8-8.3)$ & $8.6(6.0-12.0)$ & $3.2(95 \% \mathrm{CI}: 2.18,4.16) ;<0.001$ \\
Shs-cTnT (mg/L) & $0.4(0.0-0.8)$ & $0.3(0.0-0.7)$ & $0.5(0.1-1.0)$ & $0.2(95 \% \mathrm{CI}: 0.03,0.39) ; 0.086$
\end{tabular}


Table 3. Crude and multivariable adjusted OR for having significant CAD.

Unadjusted

\begin{tabular}{lccc}
\hline & OR & $\mathbf{9 5 \%}$ CI & P-value \\
\hline Resting hs-cTnT Median & 4.19 & $2.13,6.95$ & $<\mathbf{0 . 0 0 1}$ \\
Age & 1.03 & $1.00,1.06$ & $\mathbf{0 . 0 3 0}$ \\
Sex & 4.97 & $2.75,8.97$ & $<\mathbf{0 . 0 0 1}$ \\
Resting SPB & 1.02 & $1.01,1.03$ & $\mathbf{0 . 0 0 2}$ \\
Resting HR & 0.97 & $0.95,0.99$ & $\mathbf{0 . 0 0 7}$ \\
Previous revasc/MI & 1.93 & $1.16,3.19$ & $\mathbf{0 . 0 1 1}$ \\
Total cholesterol & 0.79 & $0.65,0.96$ & $\mathbf{0 . 0 1 7}$ \\
Diabetes mellitus & 1.15 & $0.86,1.55$ & 0.347 \\
Smoking & 0.61 & $0.32,1.17$ & 0.135 \\
Creatinine & 1.04 & $1.02,1.06$ & $<\mathbf{0 . 0 0 1}$
\end{tabular}


Adjusted

\begin{tabular}{lccc}
\hline & A0R & $\mathbf{9 5 \%}$ CI & P-value \\
\hline Resting hs-cTnT Median & 2.55 & $1.40,4.65$ & $\mathbf{0 . 0 0 2}$ \\
Age & 1.02 & $0.98,1.05$ & 0.422 \\
Sex & 3.29 & $1.55,6.99$ & $\mathbf{0 . 0 0 2}$ \\
Resting SBP & 1.01 & $1.00,1.03$ & 0.077 \\
Resting HR & 0.98 & $0.96,1.00$ & 0.058 \\
Previous revasc/MI & 1.32 & $0.72,2.42$ & 0.363 \\
Tot Cholesterol & 0.93 & $0.73,1.18$ & 0.547 \\
Diabetes mellitus & & & \\
Smoking & & & \\
Creatinine & 1.01 & $0.99,1.03$ & 0.356
\end{tabular}


Figure 1. ROC curves for diagnostic accuracy of significant CAD with exercise and resting hs-cTnT.

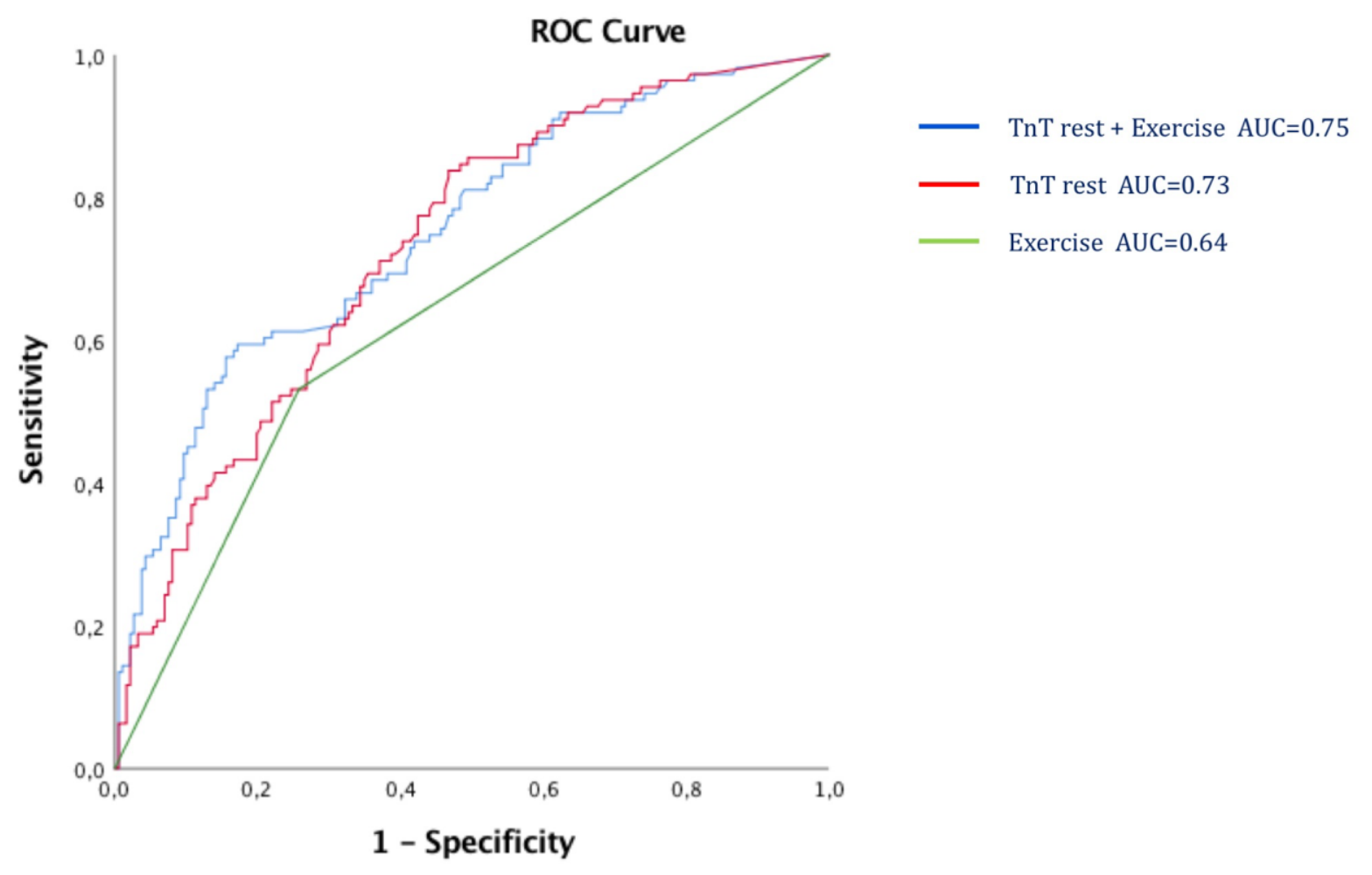


Supplementary Table 1. Exercise stress test.

\begin{tabular}{|c|c|c|}
\hline Overall $(n=297)$ & Non-sign. CAD & Sign. CAD \\
\hline & $(n=186)$ & $(n=111)$ \\
\hline
\end{tabular}

\begin{tabular}{|c|c|c|c|c|}
\hline Exercise duration (min:sec) & $10: 05 \pm 03: 46$ & $10: 22 \pm 03: 48$ & $09: 35 \pm 03: 41$ & 0.083 \\
\hline Borg scale & $17 \pm 2$ & $17 \pm 2$ & $16 \pm 2$ & 0.004 \\
\hline Workload (W) & $138 \pm 43$ & $139 \pm 44$ & $138 \pm 41$ & 0.849 \\
\hline METs & $6.9 \pm 2$ & $7.0 \pm 2$ & $6.6 \pm 2$ & 0.033 \\
\hline Resting SBP (mmHg) & $136 \pm 20$ & $133 \pm 20$ & $141 \pm 21$ & 0.002 \\
\hline Resting DBP (mmHg) & $83 \pm 11$ & $82 \pm 11$ & $84 \pm 11$ & 0.098 \\
\hline Resting HR (bpm) & $69 \pm 12$ & $71 \pm 12$ & $67 \pm 12$ & 0.007 \\
\hline Max systolic BP (mmHg) & $190 \pm 28$ & $189 \pm 28$ & $190 \pm 28$ & 0.594 \\
\hline Max diastolic BP (mmHg) & $93 \pm 13$ & $93 \pm 12$ & $94 \pm 14$ & 0.621 \\
\hline Max HR (bpm) & $140 \pm 21$ & $145 \pm 20$ & $132 \pm 20$ & $<0.001$ \\
\hline Positive test (\%) & $107(36.0)$ & $48(26)$ & $59(53)$ & $<0.001$ \\
\hline
\end{tabular}


Supplementary Table 2. Accuracy of exercise stress test and median of resting hs-cTnT for diagnosing significant CAD.

\begin{tabular}{lcccc} 
& Sensitivity & Specificity & PPV & NPV \\
\hline EST & $53 \%$ & $74 \%$ & $55 \%$ & $73 \%$ \\
Median hs-cTnT & $71 \%$ & $63 \%$ & $71 \%$ & $63 \%$ \\
EST + Median hs-cTnT & $84 \%$ & $46 \%$ & $48 \%$ & $83 \%$
\end{tabular}


Supplementary Figure 1. Patients divided according to stress test results, having significant CAD or not and resting hs-cTnT levels above or below $6.0 \mathrm{ng} / \mathrm{L}$.

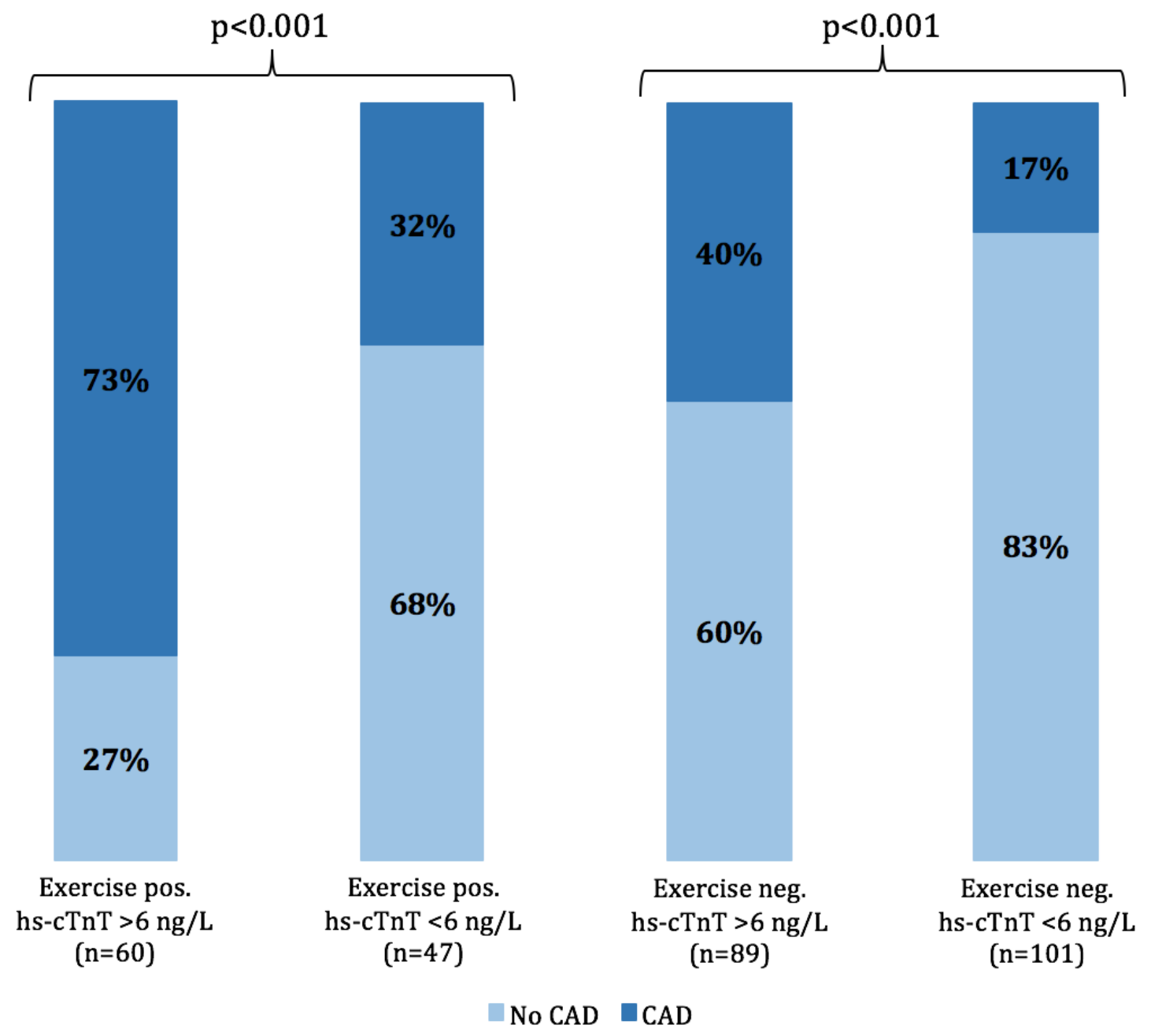

